\title{
Human epidermal keratinocytes death and expression of protein markers of apoptosis after ionizing radiation exposure
}

\author{
Sharon Wong', ${ }^{1}$, Han Hor Chor ${ }^{1}$, Sathiya Moorthy ${ }^{3}$, Chee Tian Ong1, \\ Toan Thang Phan ${ }^{1}$, Jaide Jay Lu' ${ }^{1,4}$ \\ ${ }^{I}$ Department of Medicine, National University of Singapore, Yong Loo Lin School of Medicine, Singapore. \\ ${ }^{2}$ Division of Radiation Oncology, National Cancer Centre, Singapore. \\ ${ }^{3}$ Department of Pathology, Singapore General Hospital, Singapore. \\ ${ }^{4}$ Department of Radiation Oncology, National University Cancer Institute, National University of Singapore, Singapore.
}

Received October 31, 2013; Revised November 27, 2013; Accepted November 29, 2013; Published Online December 02, 2013

\section{Original Article}

\begin{abstract}
Purpose: Knowledge of the pathophysiology of the irradiated skin is important to understand the tolerance and cosmetic response of the human skin to radiation. There are limited studies on the effect of radiotherapy dosage and fraction size in inducing apoptotic cell death in human skin. The expression of apoptotic biomarkers within a controlled population in different fractionation schemes has also never been studied. This study aims to investigate radiation induced apoptotic cell death in human skin cells after fractionated radiation exposure and the expression of unique biomarkers that reflect cell death or biology using multiplexed immunoassays. Methods: Breast skin biopsies were obtained from a single individual and divided into small pieces. Each piece was irradiated under different radiotherapy treatment fractionation schedules to a total dose of 50Gy. The irradiated skin tissues were analysed using Tunnel, immunohistochemistry and Western blot assays for expression of apoptotic keratinocytes and biomarkers (p53, p21, and PCNA). Haematoxylin and eosin (H\&E) immunostaining was performed to study the morphological changes in the skin cells. Results: Radiation is mostly absorbed by the epidermal layers and observed to damage the epidermal keratinocytes leading to the activation of apoptotic proteins. Apoptotic proteins (p53, p21 and PCNA) were confirmed to be up-regulated in radiation exposed skin cells as compared to normal skin cells with no radiation. There is strong correlation of apoptotic protein expressions with increased radiation dosage and dose fractionation. Statistical analysis with ANOVA revealed a significant increase of PCNA and p21 expression with increased radiation dosage and dose fractionation $(\mathrm{p}<$ 0.05). Immunohistochemically, $14 \%$ (range $10.71 \%$ to $17.29 \%$ ) of the keratinocytes were positive for PCNA and 22.5\% (range $18.28 \%$ to $27.2 \%$ ) for p21 after $2 \mathrm{~Gy}$ of irradiation. The most widespread, intense and uniform staining for PCNA and p21 was observed in skin that had received 50Gy of irradiation. The maximum expression of p53 (range 37.09\% to 50.91\%) was reached at $10 \mathrm{~Gy}$. Conclusion: Findings from this study will assist clinicians in predicting radiation induced skin toxicity with the current changes in radiation fractionation protocols.
\end{abstract}

Keywords: Apoptosis; Fractionated radiation therapy; Immunohistochemistry; Skin

\section{Introduction}

Radiation therapy (RT) is a commonly utilized modality for the treatment of breast cancer. It is routinely employed in breast conservation therapy. Its role as adjuvant therapy in selected patients undergoing mastectomy for stages I and II disease is currently evolving, and has become an essential

Corresponding author Dr. Sharon Wong; National Cancer Centre, Division of Radiation Oncology, 11 Hospital Drive, Singapore 169610; Email: ntrwmm@nccs.com.sg

Cite this article as:

Wong S, Chor HH, Moothy S, Ong CT, Phan TT, Lu JJ. Human epidermal keratinocytes death and expression of protein markers of apoptosis after ionizing radiation exposure. Int J Cancer Ther Oncol 2013; 1(2):01027. DOI: 10.14319/ijcto.0102.7 component of the combined modality approach for stage III disease. Postmastectomy Radiotherapy (PMRT) to the chest wall and to the regional lymphatics has shown to decrease locoregional recurrence and increase survival for women with large tumors and/or node-positive disease. ${ }^{1-2}$ However, the risk of toxicity to skin and cosmesis must be weighed against local and regional recurrences.

A review of literature suggests that the risk of radiation induced skin damage is affected not only by individual variations in response to radiation, but also by the total dose received and fractionation. ${ }^{3-7}$ There are also changes in post mastectomy radiotherapy schedules from conventional radi- 
otherapy at $50 \mathrm{~Gy}$ at 1.8 to $2 \mathrm{~Gy}$ per week to more aggressive schedules such as unconventional protocol. ${ }^{8}$ Changes in radiotherapy practice over the years are due to the recognition of the importance of fraction size, fraction number, total dose and overall time for both tumour and normal tissue reactions. However, there is very limited knowledge on the extent of radiotherapy dose and fractionation schedules in inducing cellular radiation response in the human skin.

The practice of radiotherapy would also greatly benefit from the discovery of biomarkers that correlate with symptoms and side effects pertaining to tissues within the irradiated volume. Direct or surrogate biomarkers of radiation induced toxicity could potentially give clinicians the ability to use this information to individually plan patients treatments, develop Phase I trials with molecular targeted agents in combination with RT with more confidence regarding potential side effects and/or to intervene and reduce late effects with novel therapies directed against the signalling pathway(s) involved in the late injury. ${ }^{9-10}$ However, the studies to date are limited and there is a rather weak correlation between the in-vitro and clinical data. The role of cytokines and apoptotic markers as biomarkers of radiation response to different radiation dose and fraction size has also never been studied.

In summary, this study aims to (1) look at radiation induced apoptotic cell death in human skin cells after exposure to post mastectomy treatment regimens and its relation to radiation fraction size. This study also (2) explores the proteomic expression of human skin cells as biomarkers for radiation response.

\section{Methods and Materials}

\section{Biopsies and Irradiation}

Breast skin biopsies were obtained from a single individual and divided into small pieces of $3 \mathrm{~cm}$ each, and were placed into 5 batches (Batch A, B, C, D and E). Each batch underwent a different radiotherapy treatment regimen, each receiving a total dose of 50Gy (Table 1). The human skin cell sections are irradiated daily and after each irradiation, one sample from each batch will be removed and stored in $-80^{\circ} \mathrm{C}$ freezer for biochemical analysis. Each experiment was repeated three times and average readings obtained.

TABLE 1: Experimental design

\begin{tabular}{lccc}
\hline \hline Batch & $\begin{array}{c}\text { Nos. of } \\
\text { flasks }\end{array}$ & $\begin{array}{c}\text { Dose per } \\
\text { fraction }\end{array}$ & Duration \\
\hline A-conventional & 25 & $2.0 \mathrm{~Gy}$ & 25 fractions \\
B & 20 & $2.5 \mathrm{~Gy}$ & 20 fractions \\
C & 10 & $5.0 \mathrm{~Gy}$ & 10 fractions \\
D & 5 & $10.0 \mathrm{~Gy}$ & 5 fractions \\
E & 1 & $50.0 \mathrm{~Gy}$ & 1 fraction \\
F-control & 1 & No radiation & - \\
\hline \hline
\end{tabular}

*Each batch of skin tissues were irradiated under different radiotherapy treatment regimen, each receiving a total dose of 50Gy.
The human skin cell sections are irradiated using a Gammacell@ 40 Exactor Low Dose-Rate Research Irradiator under different radiotherapy treatment schedules. Following irradiation, the skin sections were collected, fixed in $10 \%$ phosphate-buffered formalin for 10 hours at $4 \mathrm{C}$, dehydrated in ascending concentration of (50\%, 70\%, 80\%, 95\% and 100\%) ethanol solution (Merck) for 45 minutes each. This was followed by two incubations in xylene (Merck) solution for 15 minutes each. Samples were then placed onto plastic cassettes and transferred into $60^{\circ} \mathrm{C}$ liquid paraffin tank (Leica) for 30 minutes. Samples were embedded and left to solidify on the $-5^{\circ} \mathrm{C}$ cold plate. The sections were cut into $3 \mu \mathrm{m}$ thickness using a microtome (Leica) and placed onto coated glass slides.

\section{Measurement of Radiation Induced Apoptotic keratinocytes}

\section{Hematoxylin and Eosin (H\&E) staining}

Apoptotic cells are morphologically distinct due to cell shrinkage and nuclear condensation that stain darker by Haematoxylin and Eosin (H\&E). Based on these criteria, irradiated sections were stained with $\mathrm{H} \& \mathrm{E}$ and apoptotic cells were counted.

\section{TUNNEL assay}

DeadEnd TM Fluorometric TUNNEL System (Promega, Madison, WI) was used to detect apoptotic cells in tissue samples following manufacturer's protocol with some modifications. In brief, tissue sections after deparaffinization and rehydration were permeabilized with Proteinase K $(30 \mu \mathrm{g} / \mathrm{mL})$ solution, repeated fixation and wash steps. After equilibration, nucleotide mix and rTdT enzyme were added and DAPI nuclear staining dye was applied as counterstaining. The slides were mounted with cover slides and observed under x400 magnification for TUNNEL-positive cells.

\section{Immunohistochemistry (IHC) Analysis of apoptotic biomarkers}

\section{Histological procedures}

Serial paraffin sections of irradiated skin were dewaxed in xylene and then hydrated in descending concentrations of ethanol. Antigen retrieval was done by immersing the slides in $0.01 \mathrm{M}$ Tris /0.001M EDTA buffer (pH9.0) and pressure cooked for 5 minutes. All sections were cooled under running tap water for 10 minutes. The Dako Envision+ kit was used for subsequent IHC steps. Briefly, endogenous peroxidase was blocked using $3 \% \mathrm{H}_{2} \mathrm{O}_{2}$ for 30 minutes and washed in TBS-Tween 20 for 3 times. Non-specific binding was blocked using the Dako Protein Block, serum-free, for 5 minutes, followed by washes in TBS-Tween 20 for 3 times. Subsequently, sections were incubated with monoclonal anti-active p53 (Dako and BD Transduction Lab), PCNA (Santa Cruz) and P21 (Santa Cruz), in dilutions of 1:500 overnight (Table 2). 
TABLE 2: List of antibodies used in immunohistochemistry procedures

\begin{tabular}{lcccc}
\hline \hline & Antibodies & Product Code & Company & Clone \\
\hline Primary & p53 & P21020 & BD Transduction Laboratories & Monoclonal \\
& p53 & D0-7 & Dako & Monoclonal \\
& PCNA & SC-56 & Santa Cruz & Monoclonal \\
& p21 & sc-817 & Santa Cruz & Monoclonal \\
\hline Secondary & Mouse IgG, HRP-conjugated, & Dako & Polyclonal \\
& Mouse IgM, HRP-conjugated, & Dako & Polyclonal \\
& Rabbit IgG, HRP-conjugated, & Dako & Polyclonal \\
\hline \hline
\end{tabular}

After washing in TBS-Tween 20 for 3 times, the slides were incubated in secondary mouse antibody from Dako Envision+ kit for 1 hour. The slides were washed sequentially and incubated with DAB (3, 3-diaminobenzidine) for 5 minutes. Subsequently, slides were counterstained with Gill's III hematoxylin, dehydrated in ascending concentrations of ethanol, and cleared in xylene. Finally the sections were mounted using Depex (Merck). Non-immunised mouse antibody (IgG) was used for negative controls.

Briefly, tissue sectioned were screened under the microscope and evaluated under high power field (hpf, x 200). The stainings were visualized with an Olympus light microscope.

\section{Histopathological evaluation}

Sections of immunostained skin were evaluated under the microscope by an experienced pathologist (Singapore General Hospital, Singapore). The epidermal area in each biopsy sample was measured and both total and specifically stained keratinocyte nuclei were evaluated and counted. Since the number of positive cells was low in the irradiated tissue, we use the entire tissue section as reference determining the total number of keratinocyte nuclei. The difference between nonimmunoreactive (control) and immunoreactive (irradiated) keratinocyte nuclei was clear cut and cells were scored and the differences counted. The staining values are expressed as percentage of positive cells of the total number of keratinocytes evaluated with the control as a reference.

\section{Western Blot Analysis of Apoptotic biomarkers}

Protein extraction from tissue culture cells

Skin tissues were minced and lysed directly in cell lysis buffer containing 20 mM Tris-HCI (ph 7.5), 1\% Triton X-100, 100 $\mathrm{mM}$ NaCL, $0.5 \%$ Nonide P-40 and I mg/ml protease inhibitor cocktail. (Boehringer Mannheim, Mannheim, Germany). Cells were solubilized by passing solution through the pipette tip repeatedly followed by incubation on ice for 15 minutes.

Samples were then centrifuged at $4^{\circ} \mathrm{C}, 12,000 \mathrm{~g}$ for 10 minutes and the supernatant containing the proteins were collected. Total cell lysates were then subjected to Western blot analysis.

\section{Determination of protein concentrations}

Protein concentration of cell lysates (CLs) was determined using Bradford protein quantification methods according to manufacturer's instructions. A standard curve was prepared using bovine serum albumin (BSA) with concentrations ranging from $0-2000 \mu \mathrm{g} / \mathrm{ml}$ the lysis buffer. Five and ten fold dilutions were carried out for each sample and 4 repeats were done for each standard or sample. $200 \mu \mathrm{l}$ of colorimetric assay reagent was added to each well. After the 96-well plate was incubated at $37^{\circ} \mathrm{C}$ for 30 minutes, protein concentrations were assayed by measuring absorbance at $570 \mathrm{~nm}$ with a reference wavelength of $690 \mathrm{~nm}$, using a photometric plate reader (BIO-RAD, Benchmark Plus ${ }^{\mathrm{TM}}$ )

The staining intensity and reaction pattern was evaluated using the scale presented (Table 3 ).

TABLE 3: The staining intensity and pattern was evaluated using the scale below

\begin{tabular}{lc}
\hline \hline Staining Pattern & Score \\
\hline No Staining at all, or membrane staining in less than $10 \%$ of the cells & 0 \\
A faint/barely perceptible membrane staining is detected in more than $10 \%$ of the cells. & The cells are only \\
stained in part of their membrane & $1+$ \\
A weak to moderate staining of the entire membrane is observed in more than $10 \%$ of the cells & $2+$ \\
A strong staining of the entire membrane is observed in more than $10 \%$ of the cells & $3+$ \\
\hline \hline
\end{tabular}


TABLE 4: List of antibodies used in Western Blot Analysis

\begin{tabular}{ccccc}
\hline \hline & Antibodies & Product Code & Company & Clone \\
\hline Primary & p53 & P21020 & BD Transduction Laboratories & monoclonal \\
& PCNA & SC-56 & Santa Cruz & monoclonal \\
& p21 & sc-817 & Santa Cruz & monoclonal \\
\hline \hline
\end{tabular}

Table 5: Correlations between different radiation fractionation and TUNNEL staining

\begin{tabular}{|c|c|c|c|c|c|c|c|}
\hline $\begin{array}{l}\text { Total } \\
\text { Dose }\end{array}$ & $\begin{array}{c}\text { Dose } \\
\text { fractionation }^{1}\end{array}$ & $\begin{array}{c}\text { Dose } \\
\text { fractionation }{ }^{2}\end{array}$ & $\begin{array}{c}\text { Dose } \\
\text { fractionation }\end{array}$ & $\begin{array}{c}\text { Dose } \\
\text { fractionation }^{4}\end{array}$ & $\begin{array}{c}\text { Dose } \\
\text { fractionation }^{5}\end{array}$ & Pearson's R & $P$ \\
\hline 10Gy & 2Gy Day 5 & 2.5Gy Day 4 & 5Gy Day 2 & 10Gy Day 1 & --- & 0.49 & $<0.000$ \\
\hline $30 \mathrm{~Gy}$ & 2Gy Day 15 & 5Gy Day 6 & 10Gy Day 3 & --- & --- & 0.62 & $<0.000$ \\
\hline 50Gy & 2GyDay 25 & 2.5Gy Day 20 & 5Gy Day 10 & 10Gy Day 5 & 50Gy Day 1 & 0.73 & $<0.000$ \\
\hline
\end{tabular}

*Nonparametric correlations of TUNNEL positive cells with different radiation fractionation are calculated. Both Pearson's coefficient of correlation $\left(\mathrm{r}_{\mathrm{p}}\right)$ and the corresponding $\mathrm{P}$ value are shown. All statistical tests are two-sided. In all groups, there was a statistically significant correlation between TUNNEL positive cells and dose size fractionation. Radiation induced the formation of apoptotic cells and a noticeable increase was observed in skin tissues that received higher radiation dosage. These findings were confirmed by TUNNEL assay. There is also a significant $(\mathrm{p}<005)$ and strong correlation of apoptotic cells with increased fraction size.

Blocking, antibody incubation, washing and stripping Equal amounts of protein samples were denatured, separated by $12 \%$ SDS-PAGE and transferred onto nitro-cellulose membrane by semi-dry blotting. The membrane was placed protein side up and incubated for $2 \mathrm{hrs}$ in blocking solution.

Primary antibodies were diluted in 5\% milk and incubated with the membrane for $2 \mathrm{hrs}$ at room temperature. Secondary antibodies were diluted in 5\% milk and incubated with the membrane for $1 \mathrm{hr}$ at room temperature. Following primary and secondary antibody incubations (Table 4) membranes were washed 5 times each time 10 minutes with $30 \mathrm{ml}$ washing buffer and incubated in sufficient volume of buffer diluted with horseradish peroxidase (HRP)-coupled secondary antibody at room temperature for 1 hour. The membrane was washed again with washing buffer for 5 times before incubation with Western lightning plus-ECL (Perkin Elmer) for a minute, exposed on X-ray films (Konica Minolta) and developed.

\section{Computerized gel densitometry}

A Bio-Rad gel scanner and densitometer program (Gel-Pro ${ }^{\circledR}$ Analyzer version 4.5: MediaCybernetics, Maryland, USA) was used to assess concentrations of the bands obtained by Western blots. There were measured as total density units.

\section{Statistical Analysis}

Statistical Package for Social Sciences (SPSS), version 17.0 was the statistical tool used for data analysis. Nonparametric methods were used for the statistical analysis using one-way Anova (Analysis of Variance) - or Student's t-tests. A value of $\mathrm{P}<0.05$ was considered to be statistically significant.

\section{Results}

Radiation induced apoptotic keratinocyte cell death is dose and fraction size dependent

Increased Apoptotic keratinocyte cell count:

Since it has been proposed that keratinocytes damaged by ionizing irradiation undergo apoptosis, we analysed the expression of apoptotic cells in the skin tissues of the volunteer using TUNNEL assay. The skin tissues were irradiated with different fractionation schedules. (Table 5)

\section{Morphological changes of radiation induced apoptotic keratinocytes}

Results showed that radiation is mostly absorbed by the epidermal layers and observed to damage the epidermal keratinocytes leading to apoptosis. These cells had characteristic dyskeratotic apoptotic cells with pyknotic nuclei in the irradiated sections. HE staining on control sections demonstrated normal epidermal morphology (Figure 1A) while irradiated sections demonstrated strong evidence of cellular damage caused by ionizing radiation (Figure 1B-D).

The keratinocytes were enlarged with cytological atypia Figure 2. The epidermis in the irradiated skin was thin, containing only one to three layers of keratinocytes and in some tissues; the epidermis layer was also detached from the dermis layer Figure 3. 


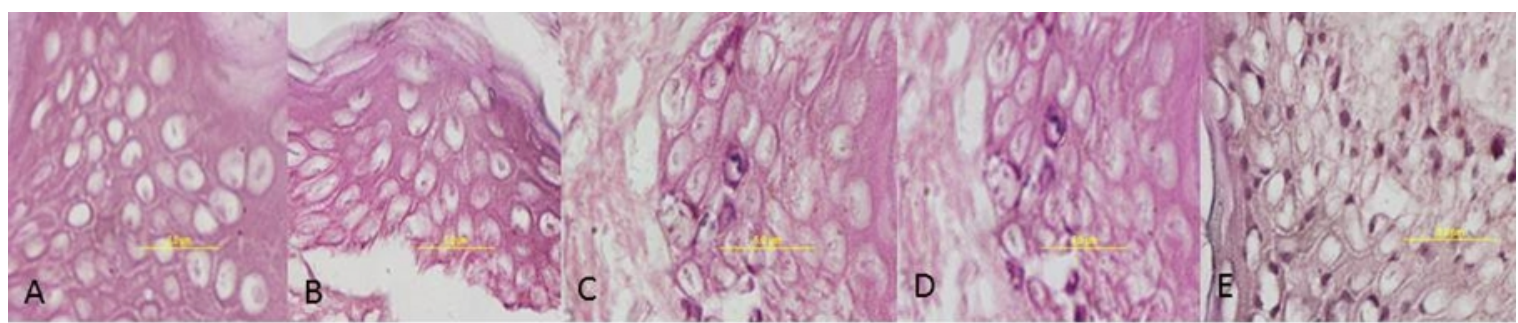

Dose series of Irradiated skin. Sections were stained with $\mathrm{HE}$.

\section{A- Control, B- 2Gy, C-5Gy, D-10Gy, E- 50Gy}

FIG. 1: Morphological evaluation

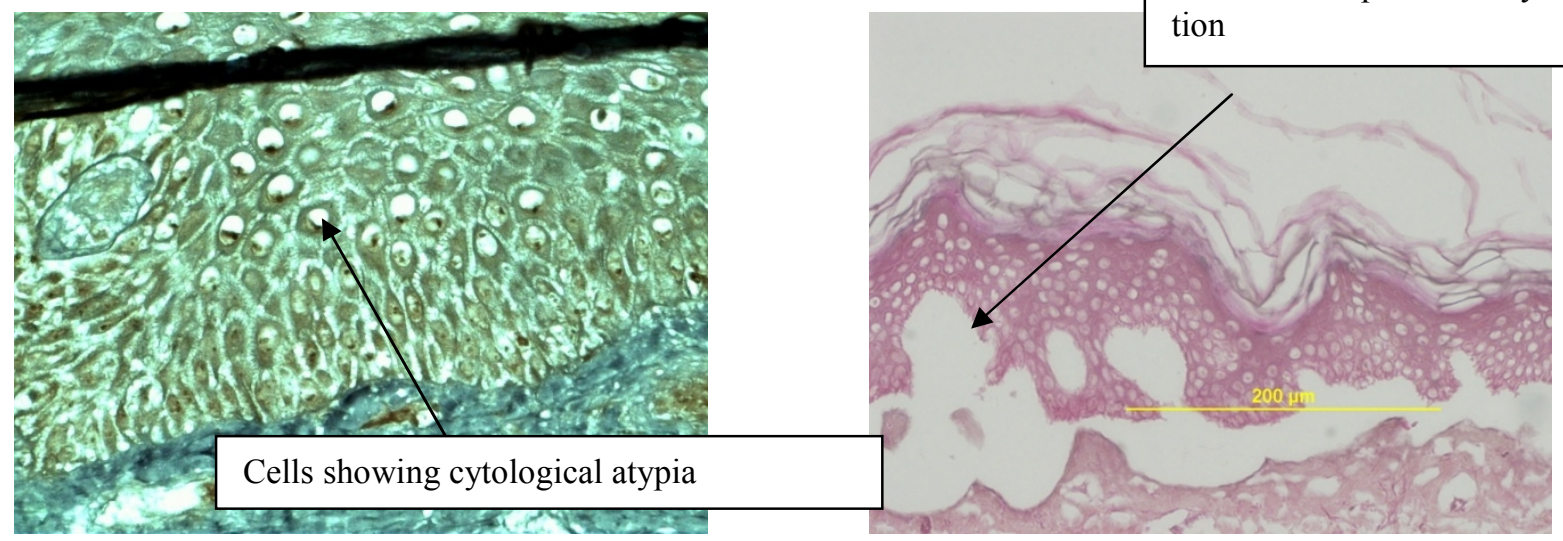

FIG. 2 (left): Shows morphological changes in cell after layer due to the irradiation.

FIG. 3 (right): HE slide showing a detached epidermis radiation exposure.

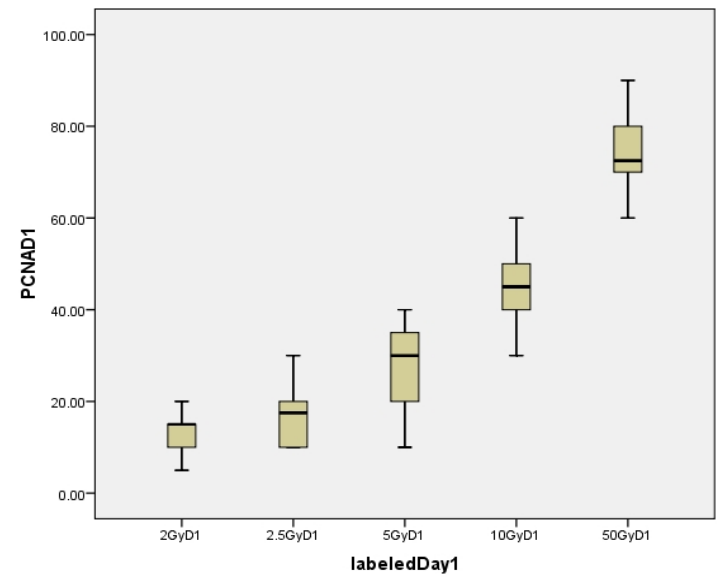

FIG. 4: The boxplot for PCNA immunoreactitivity in different radiation dosage starting from $2 \mathrm{~Gy}$ to $50 \mathrm{~Gy}$. Bars represent the range within the different radiation dosage as indicated.

Expression of apoptosis related marker proteins with irradiation and this is dose and fraction size dependent

Accumulation of p53, PCNA and p21 proteins with increasing dose

We analyzed sections of paraffin-embedded skin biopsies of the volunteer for p53, PCNA and p21 apoptotic related

FIG. 5: The boxplot for p21 immunoreactitivity in different radiation dosage starting from $2 \mathrm{~Gy}$ to $50 \mathrm{~Gy}$. Bars represent the range within the different radiation dosage as indicated.

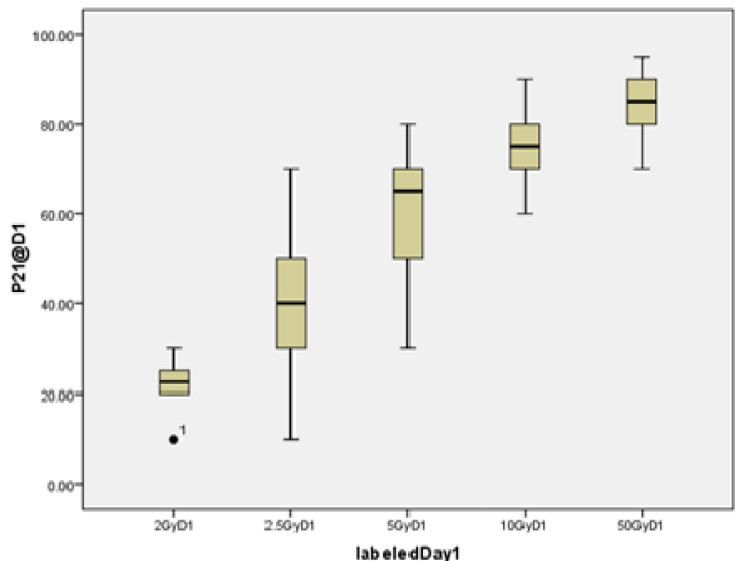

marker proteins using western blot and immunohistochemistry analysis. Hardly any apoptotic positive cells were seen in the control epidermis before radiation. Skin tissues were irradiated with 2, 2.5, 5, 10 and 50 Gray. Apoptotic proteins (PCNA, P21) were confirmed to be up-regulated in radiation exposed skin cells as compared to normal skin cells with no radiation. There is strong correlation of apoptotic protein expressions with increased radiation dosage. (Figures 4-5) 
In contrast, $\mathrm{P} 53$ expression started to increase after 2Gy of radiation up to a maximum dose of $10 \mathrm{~Gy}$ (Figure 6). At $2 \mathrm{~Gy}$, a higher number of cells faintly positive for p53 extended from the suprabasal layer to the stratum granulosum. At more superficial locations, these cells became strongly stained by anti-p53. The maximum expression of p53 (median $=44 \%$, range $37.09 \%$ to $50.91 \%$ ) was reached at $10 \mathrm{~Gy}$ after irradiation exposure, and in most cases the p53 immunoreactivity appeared to be concentrated within the keratinocyte nuclei. At higher radiation dosage (> 10Gy), the epidermal sections showed a downward trend in the expression of P53. The irradiated keratinocyte nuclei appeared to be p53 negative or only faintly stained at $50 \mathrm{~Gy}$ (median $=7 \%$, range $5.15 \%$ to $8.85 \%$ ). Western blot analysis revealed similar results.

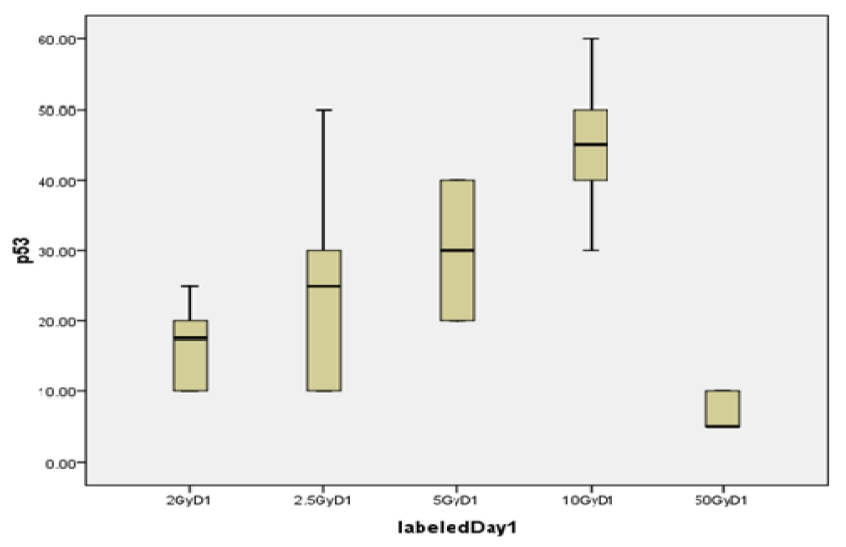

FIG. 6: The boxplot for p53 immunoreactitivity in different radiation dosage starting from $2 \mathrm{~Gy}$ to $50 \mathrm{~Gy}$. Bars represent the range within the different radiation dosage as indicated.

\section{Morphological changes of PCNA, P21 and P53 with increas- ing dose}

Results unequivocally demonstrated that, in response to DNA damage, PCNA (Figure 7) and p21 (Figure 8) are up-regulated for cell cycle arrest to facilitate DNA repair.

As for p53, we hardly detected any cells stained with anti-p53 antibody in the control slide (Figure 9). P53 expression started to increase after $2 \mathrm{~Gy}$ of irradiation and maximal expression of p53 was reached at 10Gy. Thereafter irradiated cells appeared to be p53 negative or only faintly stained at $50 \mathrm{~Gy}$.

Accumulation of p53, PCNA and p21 proteins with increasing fraction size

Subsequently, we tested by immunohistochemistry the expression of marker proteins of apoptosis, namely p53, P21 and PCNA with different radiation fractionations.

Repeated measures of ANOVA revealed that the effects of different radiation fractionation (2Gy, 2.5Gy, 5Gy, 10Gy and $50 \mathrm{~Gy}$ ) on human skin cells were significantly different from each other $(\mathrm{p}<0.05)$. There is a positive and significant correlation (Pearson's correlation $\mathrm{r}$ ) between radiation fractionation and apoptotic protein expression (PCNA, p21 and P53), measured at different dosage. This correlation is strongest at higher dose (Table 6 and 7).

In summary, we have checked for associations between cytokine expression, radiation dose and type of fractionation regimen, given that both radiation and proteins expression are important variables in mediating radiation response. Results have shown that PCNA and p21 levels were significantly associated with dose and treatment regimen, while elevation of p53 was significant only up to $10 \mathrm{~Gy}$. These results were supported by western blot findings.

\section{Western blot analysis revealed elevated levels of PCNA and P21 proteins in Irradiated keratinocytes and this is dose and fraction size dependent}

Little or no immunoreactive band specific for p53, PCNA and p21 protein was observed in the lysates of non-irradiated control keratinocytes. Irradiated cell lysates revealed an accumulation of p53 protein which was detected at 2Gy and peaked at 5Gy. After 5Gy, the expression level slightly declined and was not detectable at 50Gy (Figure 10).

Regarding p21 and PCNA, a weak band was seen in control keratinocytes. Overexpression of p21 and PCNA protein was seen which increases with radiation dosage and fractionation (Figures 11-14).

This is one of the few studies that evaluated the difference in cellular response due to minor variations in the protocols of fractionation radiotherapy. For the evaluation of the effect of changes in dose/fraction, the human skin cells irradiated with the same total dose of 10Gy, $30 \mathrm{~Gy}$ and 50Gy were grouped according to the dose/fraction given. No significant difference in apoptotic cell death between groups of samples irradiated by dose fraction of $2 \mathrm{~Gy}$ and $2.5 \mathrm{~Gy}$ could be detected. However, irradiation with a higher dose/fraction of $5 \mathrm{~Gy}$ and $10 \mathrm{~Gy}$ resulted in a very significant elevation of mean \% apoptotic cell death expression. Moreover, significant changes were also found at much lower total dose of 30Gy, where the treatment was given with a high dose/fraction of about $5 \mathrm{~Gy}$. Our results demonstrate that the increase in dose of radiation per fraction had much more impact on radiation induced apoptosis than elevation in the total dose, which may result in worst skin outcome. Our result is similar to previous study by Archambeau ${ }^{11}$ who evaluated the effect of changes in dose/fraction using a swine skin model. Following irradiation there is a dose-dependent loss of basal cells. The fields treated with 2Gy daily dose fractions show no change in the basal cell density until total doses of 20-25Gy are delivered. This period is followed by 
increasing cell loss up to 50Gy and necrosis is produced. A separate study by Raphael ${ }^{12}$ looked at the effect of radiation fraction size on skin viscoelasticity. They also found that the increase in dose of radiation per fraction greatly increased skin stiffness. There is an intimate relationship between radiation-dose fractionation and clinical outcome of radiation therapy.

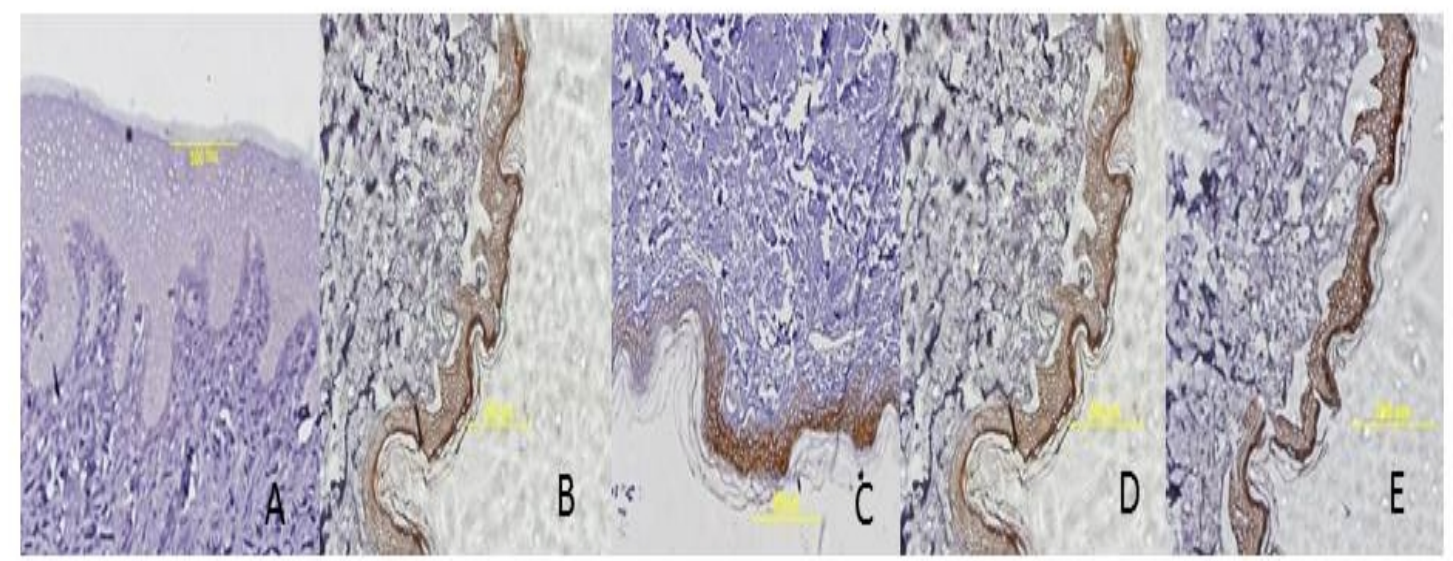

FIG. 7: Dose series of Irradiated skin. Sections were stained with monoclonal anti-PCNA; A-Control, B-2Gy, C-5Gy, D-10Gy, E-50Gy

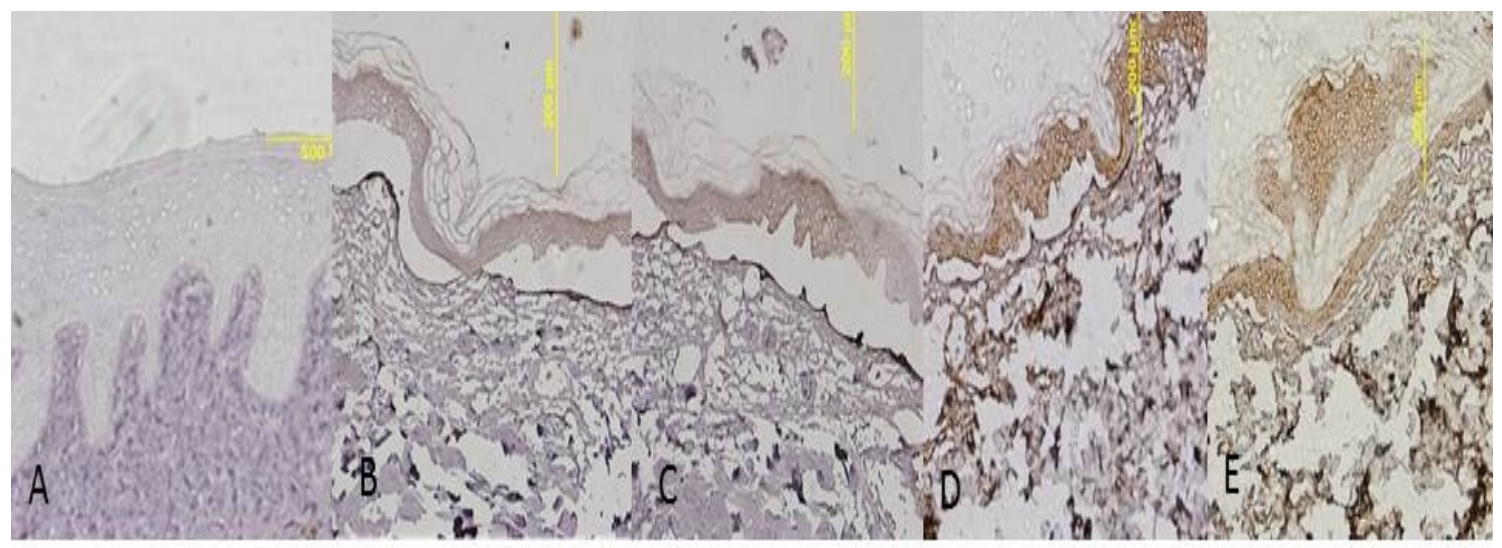

FIG. 8: Dose series of Irradiated skin. Sections were stained with monoclonal anti-p21; A-Control, B- 2Gy, C-5Gy, D-10Gy, E-50Gy

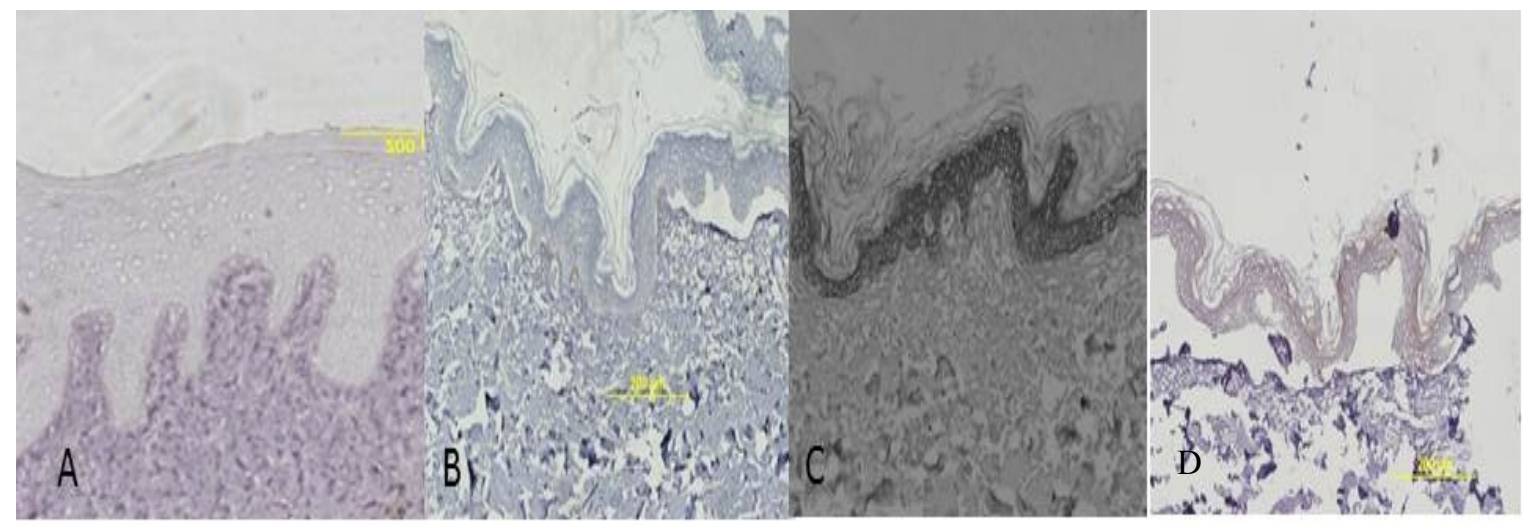

FIG. 9: Dose series of Irradiated skin. Sections were stained with monoclonal anti-p53; A-Control, B- 2Gy, C-10Gy, D-50Gy 
TABLE 6: Correlations between different radiation fractionation and PCNA expression

\begin{tabular}{|c|c|c|c|c|c|c|c|}
\hline $\begin{array}{l}\text { Total } \\
\text { Dose }\end{array}$ & $\begin{array}{c}\text { Dose } \\
\text { fractionation }^{1}\end{array}$ & $\begin{array}{c}\text { Dose } \\
\text { fractionation }{ }^{2}\end{array}$ & $\begin{array}{c}\text { Dose } \\
\text { fractionation }{ }^{3}\end{array}$ & $\begin{array}{c}\text { Dose } \\
\text { fractionation }^{4}\end{array}$ & $\begin{array}{c}\text { Dose } \\
\text { fractionation }^{5}\end{array}$ & Pearson's R & $P$ \\
\hline $10 \mathrm{~Gy}$ & 2Gy Day 5 & 2.5Gy Day 4 & 5Gy Day 2 & 10Gy Day 1 & -- & 0.59 & $<0.000$ \\
\hline $30 \mathrm{~Gy}$ & 2Gy Day 15 & 5Gy Day 6 & 10 Gy Day 3 & --- & --- & 0.61 & $<0.002$ \\
\hline 50Gy & 2GyDay 25 & 2.5Gy Day 20 & 5Gy Day 10 & 10Gy Day 5 & 50Gy Day 1 & 0.66 & $<0.000$ \\
\hline
\end{tabular}

TABLE 7: Correlations between different radiation fractionation and p21 expression

\begin{tabular}{|c|c|c|c|c|c|c|c|}
\hline $\begin{array}{l}\text { Total } \\
\text { Dose }\end{array}$ & $\begin{array}{c}\text { Dose } \\
\text { fractionation }^{1}\end{array}$ & $\begin{array}{c}\text { Dose } \\
\text { fractionation }{ }^{2}\end{array}$ & $\begin{array}{c}\text { Dose } \\
\text { fractionation }^{3}\end{array}$ & $\begin{array}{c}\text { Dose } \\
\text { fractionation }\end{array}$ & $\begin{array}{c}\text { Dose } \\
\text { fractionation }^{5}\end{array}$ & Pearson's R & $P$ \\
\hline 10Gy & 2Gy Day 5 & 2.5Gy Day 4 & 5Gy Day 2 & 10Gy Day 1 & --- & 0.657 & $<0.000$ \\
\hline $30 \mathrm{~Gy}$ & 2Gy Day 15 & -- & 5 Gy Day 6 & 10Gy Day 3 & 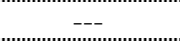 & 0.523 & $<0.002$ \\
\hline 50Gy & 2GyDay 25 & 2.5Gy Day 20 & 5Gy Day 10 & 10Gy Day 5 & 50Gy Day 1 & 0.634 & $<0.000$ \\
\hline
\end{tabular}

*Tables 6 \& 7: Nonparametric correlations of PCNA and P21 immunoreactive cells with different radiation fractionations are calculated. Both

Pearson's coefficient of correlation $\left(\mathrm{r}_{\mathrm{p}}\right)$ and the corresponding P value are shown. All statistical tests are two-sided.

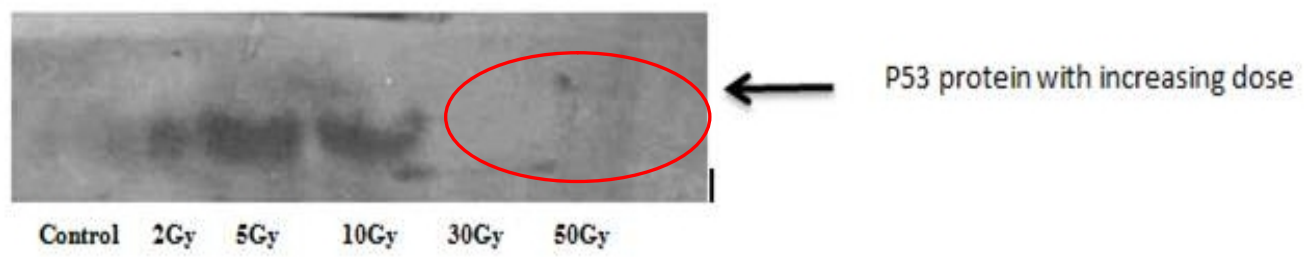

FIG.10: Little or no immunoreactive band specific for p53 was observed at dose above 10Gy

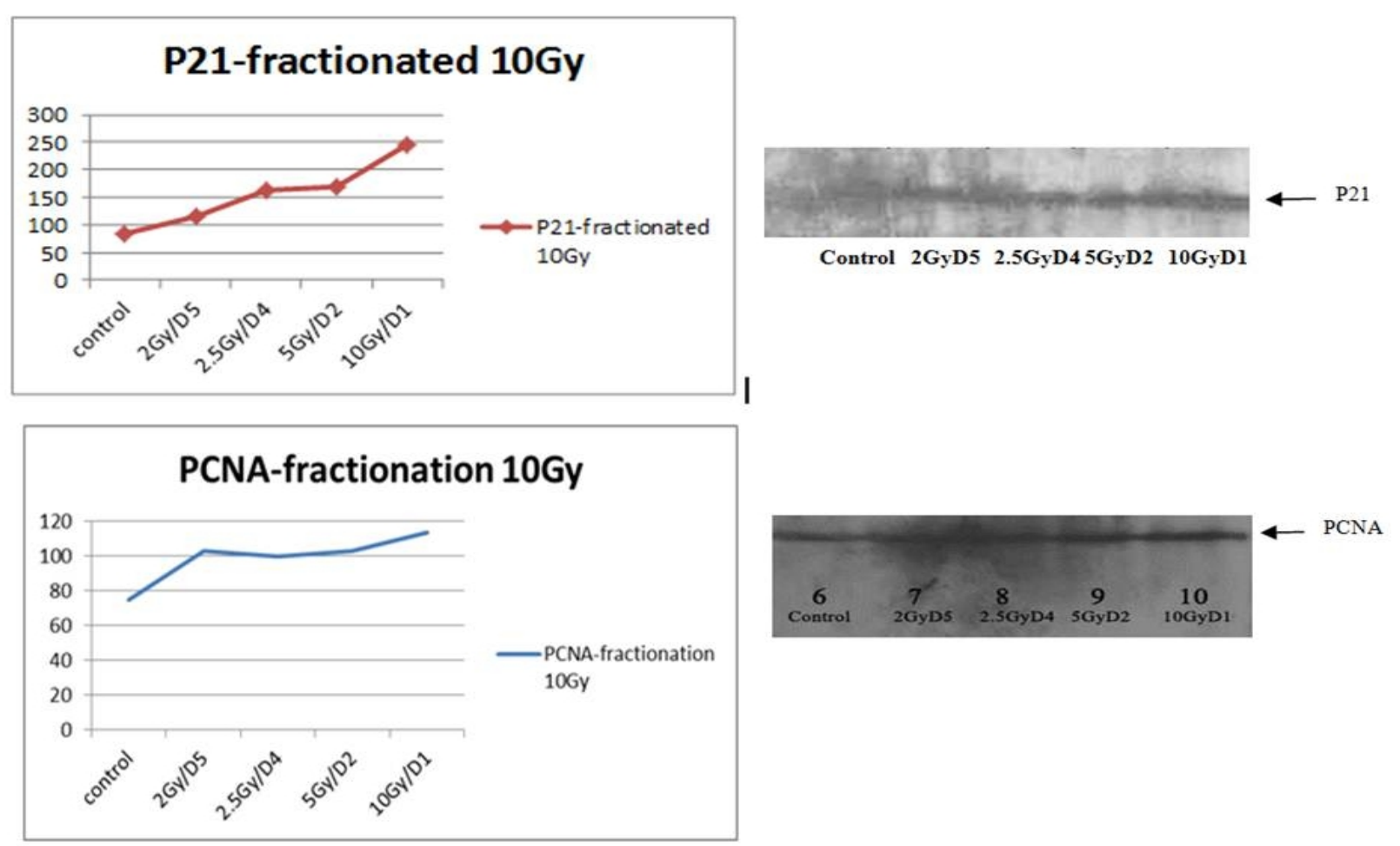

FIG.11 (top) and FIG. 12 (bottom): Western blot analysis of P21 and PCNA reveals a higher level of protein with increasing fraction size at 10Gy 

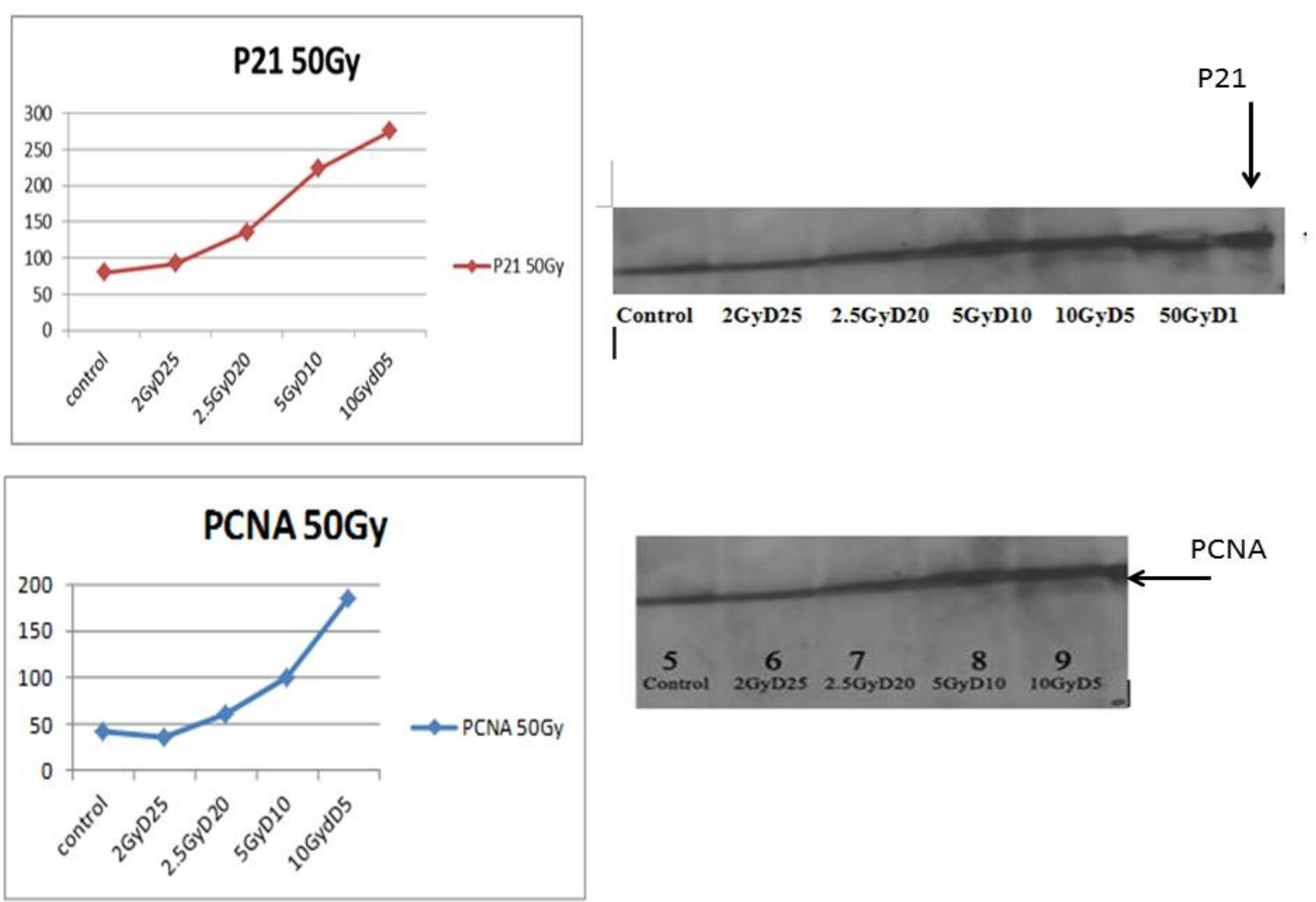

FIG.13 (top) and FIG. 14 (bottom): Western blot analysis of P21 and PCNA reveals a higher level of protein with increasing fraction size at 50Gy

\section{Discussion}

The practice of radiotherapy would greatly benefit from the understanding of apoptosis and its associated biomarkers that correlate with radiation dose and its side effects pertaining to tissues within the irradiated volume. This forms the basis of our study.

In this study we demonstrated dose-dependent keratinocyte apoptosis following irradiation that was associated with activation of apoptosis regulating biomarkers. We demonstrated that increasing radiation dosage led to increased keratinocyte death in human skin. Our results are in agreement with the findings of previously reported studies showing a dose related induced apoptosis using swine skin ${ }^{11}$ and human cochlear cells. ${ }^{13}$ Morphological evaluation was consistent with our findings. HE staining revealed an increased in the number of apoptotic cells with condensed nuclei surrounded by a bright halo. These morphological changes were similar to what has been described in Ponten F. ${ }^{14}$ The keratinocytes were enlarged with cytologic atypia.

The relationship between the total dose of radiation and fractionation of the dose given is also crucial in radiotherapy with respect to the development of radiation induced effects in normal tissues. This elevates the therapeutic ratio and enables efficient tumour control while decreasing both early and late radiation effects in normal tissues. ${ }^{12}$

Cellular damage caused by ionizing radiation induces production and recruitment of specific proteins that have the potential to predict radiation induced toxicity in tissues within the irradiated volume. ${ }^{15-16}$ Our interest in these proteins expression during Radiation Therapy is based in part on Rubin ${ }^{17}$ and colleagues' description of a cytokine-linked continuum of early and late radiation responses. Rubin et al showed in murine and clinical lung models that radiation induced fibrosis is progressive after treatment and begins immediately following lung irradiation. Levels of IL-1, TGF beta and TNF alpha were observed to increase in lung tissue immediately after radiation exposure, however clinically appreciable lung fibrosis may occur months later. A number of clinical studies have also shown that cytokines are elevated during and after Radiotherapy for a variety of malignancies. ${ }^{10,}{ }^{18-19}$ Using this as a feasibility study, we measured the cytokines protein levels of p53, PCNA and p21 in human breast skin cells after receiving post-mastectomy radiotherapy treatment regimens as biomarkers for radiation 
response. The radiation response of cytokines within a controlled population with the same dose but in different fraction size has never been studied before. Thus this is one of the first studies of its kind on cytokines expression in human breast skin after exposure to fractionated radiation.

Statistical analysis with ANOVA revealed a significant increase of PCNA and p21 expression with increased radiation dosage $(\mathrm{p}<0.05)$ and with different radiation fraction size $(p<0.05)$. Our results showed that cytokines are up-regulated in response to radiation; and this is dependent on radiation dosage and fraction size. This is in agreement with previous studies that shown that these cytokines are radiation-inducible cytokine. Fredrik Ponten et al ${ }^{14}$ investigated levels of p53 and p21 in skin exposed to different types of radiation. Results show an early onset of increased p53 levels and a correlation between p53 and p21 was evident. In other studies, p53, PCNA and p21 were induced in hairless mouse epidermis following UVB radiation ${ }^{20}$, human skin response to $\mathrm{x}$-ray treatment with release of p53 ${ }^{21}$ and induced expression of $\mathrm{p} 21$ and PCNA proteins in UVB irradiated human epidermis. ${ }^{22}$ However, drawbacks of these studies were the use of mice epidermis and UVB radiation and included a range of radiation dose. In our study, these factors were closely controlled. Human breast epidermis from a single donar and 50Gy of $6 \mathrm{MV}$ ionizing radiation were used.

Results from our studies show predictable patterns of cytokine expression. PCNA and P21 levels elevated gradually from $2 \mathrm{~Gy}$ to $10 \mathrm{~Gy}$, followed with a sharp increase at $50 \mathrm{~Gy}$. On the other hand, p53 was elevated at $2 \mathrm{~Gy}$ and persisted until 10Gy, and then returned to baseline values. This pattern was also consistent with increasing fraction size. Not only did this study demonstrate the early and persistent elevation of cytokine production following irradiation, but it also determined consistent patterns of cytokines expression. A similar pattern of cytokine expression was observed in murine studies. Rubin ${ }^{17}$ and colleagues studied cytokine induction in mice receiving thoracic irradiation. They found several cytokines were longitudinally elevated with consistent patterns between mice.

One question arising from our results is why p53 did not exhibit similar pattern with PCNA and p21 given that all are apoptotic inducing cytokines. P53 plays a crucial role in the protection against DNA damage allowing cell cycle arrest, DNA repair or apoptosis by transcriptional activation of p53 related genes such as $\mathrm{p} 21$ and Bax. ${ }^{23}$ Ionizing radiation produces double and single strand DNA breaks. Cells respond to DNA photoproducts and DNA breaks by accumulation of functionally active $\mathrm{p} 53$ proteins.

Upon exposure to radiation, p53 is known to sense DNA damaged and to subsequently halt the cell cycle in the G1 phase, providing time for DNA repair. If the damage to the genome is too serious and repair likely to fail, p53 acts as an inducer of apoptosis. ${ }^{14}$ Consistent with this report, we observed that these proteins are up-regulated in response to irradiation-caused DNA damage leading to cell cycle arrest to allow enough time for DNA repair. This observation is consistent with similar study in animal model showing stimulatory effect of UV radiation on the protein levels of p53 and downstream effector p21. ${ }^{20}$ In a separate time-dose study by Peter Mab et al ${ }^{21}$ human skin was exposed to UVB radiation and p53 expression was detection. P53 expression started to increase $3 \mathrm{~h}$ after irradiation and reached a maximum at $12 \mathrm{~h}$ after UVB exposure. However, epidermal sections at $36 \mathrm{~h}$ appeared to be $\mathrm{p} 53$ negative or only faintly stained. This seem to suggest $\mathrm{p} 53$ has a short half life of less than $24 \mathrm{~h}$ and is time -dose dependent. However, more studies are needed in the future to further support this anticipation, Further in this study, the radiation induced p21 protein was detected much sooner than PCNA, correlating well with the expression of p53. It seems likely that up regulation of $\mathrm{p} 21$ could have been mediated via $\mathrm{p} 53$ pathway by irradiation. The different time course of the expression also supports this hypothesis.

PCNA is implicated in DNA replication and repair by forming a sliding platform that could mediate the interaction of numerous proteins with DNA. ${ }^{24}$ It is known that p21 binds to PCNA and inhibits PCNA function in DNA replication. ${ }^{25}$ Therfore PCNA is regarded as an important target for p21 as well as reliable biomarker for cell proliferation. It has been observed that p53/p21 signal transduction pathway plays a significant role in the regulation of the PCNA response to ionizing radiation. ${ }^{26}$ In view of the high affinity of PCNA to DNA strand breaks due to the ionizing radiation ${ }^{27}$, it is logical to expect the rapid recruitment of PCNA to ionizing radiation induced strand breaks. In this study, we have demonstrated the induction of PCNA after radiation in human skin cells and this induction is dose and fraction size dependent.

The evidence from our study and clinical literature demonstrate a significant dose and fraction size response during RT. They also support the molecular rationale that increased cytokines expression may serve as indicators of increased normal tissue toxicity. The clinical research environment presents a number of unique challenges to prospective proteomics studies from sample collection to processing, storage and analysis. There are many sources of error (delays in sample collection or processing, misinterpretation of standard operating procedures (SOP) and so on) that can introduce a myriad of confounding variables, which in turn will render the resulting proteomic data inconclusive. We have considered these issues in details and study designs were carefully drawn out to minimize the influence of extraneous environmental factors that may affect our conclusions from these data. In addition our principal data were derived from a single 
volunteer, thus avoided interindividual variations in the responses to DNA damage.

Another limitation of our work is the small sample size and limited apoptotic markers identified in skin relating to postmastectomy radiotherapy. Future studies with bigger sample sizes are warranted to determine the cytokine changes after radiation exposure and how these relate to normal tissue radiation toxicity with greater certainty.

\section{Conclusion}

In summary, our results demonstrate the specific up-regulation of a number of apoptotic related marker proteins after irradiation and suggest that premature keratinocyte elimination occurs via apoptosis. Results also demonstrated that their increase expression or activation occurs in relation to radiation dose and fraction size. While total dose is critical in determining the severity of skin toxicity, skin effects are more severe following fractionations schedules that employ daily dose fractions of 5Gy or higher than follow dose schedules using 2-2.5Gy daily.

More importantly, based on our findings, there is a potential for the use of anti-apoptotic strategies which could be targeted at various regions of the apoptotic pathway to prevent radiation induced skin toxicity. Access of anti-apoptotic factors to the skin could possibly be delivered topically. The use of such a target-specific approach would minimize the systemic side effects of anti-apoptotic treatments. However, the mechanisms regulating the increase in cytokines in vivo in response to cellular damage and their role need additional study.

Taken together, all of the experiments discussed in this study lay the groundwork for future larger scale clinical trials within our institution and in collaboration with other clinical centers. Protein markers have potential as proxy indicators of radiation sensitivity and exposure. It is hoped that the preliminary work presented here will contribute to the identification of protein biomarkers that contributes not only to the advancement of radiation biology in the long-term, but allows us to make a difference in the lives of cancer patients. The very high incidence of breast cancer partially attributable to the ageing of their populations and the increasing use of surgery and postoperative radiotherapy for its treatment make the above type of study of special interest, with the side effects of radiotherapy an increasingly important issue.

\section{Conflict of interest}

The authors declare that they have no conflicts of interest. The authors alone are responsible for the content and writing of the paper.

\section{References}

1. Overgaard M, Jensen MB, Overgaard J, Hansen PS, Rose C, et al. Postoperative radiotherapy in high-risk postmenopausal breast-cancer patients given adjuvant tamoxifen: Danish Breast Cancer Cooperative Group DBCG 82c randomised trial. Lancet 1999; 353:1641-1648.

2. Ragaz J, Jackson SM, Le N, Plenderleith IH, Spinelli JJ, et al. Adjuvant radiotherapy and chemotherapy in node-positive premenopausal women with breast cancer. N Engl J Med 1997; 337: 956-962.

3. Turesson, I. Individual variation and dose dependency in the progression rate of skin telangiectasia. Int J Radiat Oncol Biol Phys 1990; 19: 1569-1574.

4. Turesson I, Nyman J, Holmberg E, et al. Prognostic factors for acute and late skin reactions in radiotherapy patients. Int J Radiat Oncol Biol Phys 1996; 36; 1065-1075.

5. Tucker SL, Turesson I, Thames HD. Evidence for individual differences in the radiosensitivity of human skin. Eur J Cancer 1992; 28A: 1783-1791.

6. Bernier J, Thames HD, Smith CD, Horiot JC. Tumor response, mucosal reactions and late effects after conventional and hyperfractionated radiotherapy. Radiother Oncol 1998; 47: 137-143.

7. Rojas A, Joiner MC. The influence of dose per fraction on repair kinetics. Radiother Oncol 1989; 14: 329-336.

8. Vicini FA, Baglan KL, Kestin LL, Mitchell C, Chen PY, Frazier RC, Edmundson G, Goldstein NS, Benitez P, Huang RR, Martinez A. Accelerated treatment of breast cancer. J Clin Oncol 2001; 19:1993-2001.

9. Jager JJ, Jansen RL, Arends JW. Clinical relevance of apoptotic markers in breast cancer not yet clear. Apoptosis 2002; 7: 361-365.

10. Menard C, Johann D, Lowenthal M, Muanza T, Sproull M, Ross S, et al. Discovering clinical biomarkers of ionizing radiation exposure with serum proteomic analysis. Cancer Res 2006; 66: 1844-1850.

11. Archambeau JO, Pezner R, Wasserman T. Pathophysiology of irradiated skin and breast. Int $\mathrm{J}$ Radiat Oncol Biol Phys 1995; 31:1171-1185.

12. Gorodetsky R, Lotan C, Piggot K, Pierce LJ, Polyansky I, et al. Late effects of dose fractionation on the mechanical properties of breast skin following post-lumpectomy radiotherapy. Int $J$ Radiat Oncol Biol Phys 1999; 45:893-900.

13. Low WK, Tan MG, Sun L, Chua AW, Goh LK, Wang DY. Dose-dependant radiation-induced apoptosis in a cochlear cell-line. Apoptosis 2006; 11:2127-2136. 
14. Ponten F, Lindman H, Bostrom A, Berne B, Bergh J. Induction of p53 expression in skin by radiotherapy and UV radiation: a randomized study. J Natl Cancer Inst 2001 ;93:128-133.

15. Faulhaber O, Bristow RG. Basis of cell kill folowing clinical radiotherapy, In M. Sluyser(ed), Application of Apoptosis to Cancer Treatment. Springer 2005; 293-320.

16. Hagan $M$, Yacoub A, Dent P. Ionizing radiation causes a dose-dependent release of transforming growth factor alpha in vitro from irradiated xenografts and during palliative treatment of hormone-refractory prostate carcinoma. Clin Cancer Res 2004; 10: 5724-5731.

17. Rubin P, Johnston CJ, Williams JP, McDonald S, Finkelstein JN. A perpetual cascade of cytokines postirradiation leads to pulmonary fibrosis. Int $J$ Radiat Oncol Biol Phys 1995; 33:99-109.

18. Okunieff P, Chen Y, Maguire DJ, Huser AK. Molecular markers of radiation-related normal tissue toxicity. Cancer Metastasis Rev 2008; 27:363-374.

19. Kovacs CJ, Daly BM, Evans MJ, Johnke RM, Lee TK, et al. Cytokine profiles in patients receiving wide-field + prostate boost radiotherapy (xRT) for adenocarcinoma of the prostate. Cytokine 2003; 23:151-63.

20. Gu M, Dhanalakshmi S, Singh RP, Agarwal R. Dietary feeding of silibinin prevents early biomarkers of UVB radiation-induced carcinogenesis in SKH-1 hairless mouse epidermis. Cancer Epidemiol Biomarkers Prev 2005; 14:1344-9.
21. Mass P, Hoffmann K, Gambichler T, Altmeyer P, Mannherz HG. Premature keratinocyte death and expression of marker proteins of apoptosis in human skin after UVB exposure. Arch Dermatol Res 2003; 295:71-79.

22. Ahmed NU, Ueda M, Ichihashi M. Induced expression of p16 and p21 proteins in UVB-irradiated human epidermis and cultured keratinocytes. J Dermatol Sci 1999; 19:175-181.

23. Murphy M, Mabruk MJ, Lenane $P$, Liew A, McCann P, Buckley A, et al. The expression of p53, p21, Bax and induction of apoptosis in normal volunteers in response to different doses of ultraviolet radiation. $B r \quad J$ Dermatol 2002; 147:110-107.

24. Warbrick E. The puzzle of PCNA's many partners. Bioessays 2000; 22;997-1006.

25. Fotedar R, Bendjennat $M$, Fotedar A. Role of p21WAF1 in the cellular response to UV. Cell Cycle 2004; 3:134-137.

26. Wenz F, Azzam EI, Little JB. The response of proliferating cell nuclear antigen to ionizing radiation in human lymphoblastoid cell lines is dependent on p53. Radiat Res 1998; 149:32-40.

27. Moggs JG, Grandi P, Quivy JP, Jonsson ZO, Hubscher U, Becker PB, Almouzni G. A CAF-1-PCNA-mediated chromatin assembly pathway triggered by sensing DNA damage. $\mathrm{Mol}$ Cell Biol 2000; 20:1206-18. 